\title{
Controllable Soil Degradation Rate of 5-Substituted Sulfonylurea Herbicides as Novel AHAS Inhibitors
}

\author{
Shaa Zhou, ${ }^{*}{ }^{\dagger, *}$ Fan-Fei Meng, ${ }^{\star}$ Xue-Wen Hua, ${ }^{\S}$ Yong-Hong Li, ${ }^{\ddagger}$
}

Bin Liu, ${ }^{\ddagger}$ Bao-Lei Wang, ${ }^{\star}$ Jie Chen,${ }^{\dagger}$ An-Liang Chen,${ }^{\dagger}$ Zheng-Ming Li ${ }^{*}$,

${ }^{\dagger}$ Collaborative Innovation Center of Green Pesticide, National Joint Engineering Laboratory of Biopesticide Preparation, Zhejiang A\&F University State Key Laboratory of Subtropical Silviculture, School of Forestry and Biotechnology, Zhejiang A \& F University, Hangzhou 311300, Zhejiang, China

${ }^{\ddagger}$ State Key Laboratory of Elemento-Organic Chemistry, Collaborative Innovation Center of Chemical Science and Engineering (Tianjin), College of Chemistry, Nankai University, Tianjin 300071, China

${ }^{\S}$ College of Agriculture, Liaocheng University, Liaocheng 252000, China

* Address correspondence to this author at State Key Laboratory of Elemento-Organic Chemistry, Nankai University, No. 94, Weijin Road, Nankai District, Tianjin 300071, China (telephone: +86 022-23503732; Fax: +86 022-23503732; e-mail: nkzml@vip.163.com)

*Address correspondence to Shaa Zhou at School of Forestry and Biotechnology, Zhejiang A \& F University, No. 666, Wusu st., Linan District, Hangzhou 311300 , China (telephone: +86 0571-61073177; e-mail: xiaomizhoulove@163.com) 


\section{Supporting Information}

Data 1 Figure S1-4 and Table S1-9

Data 2 Physical properties of the target compounds

\section{Data 1}
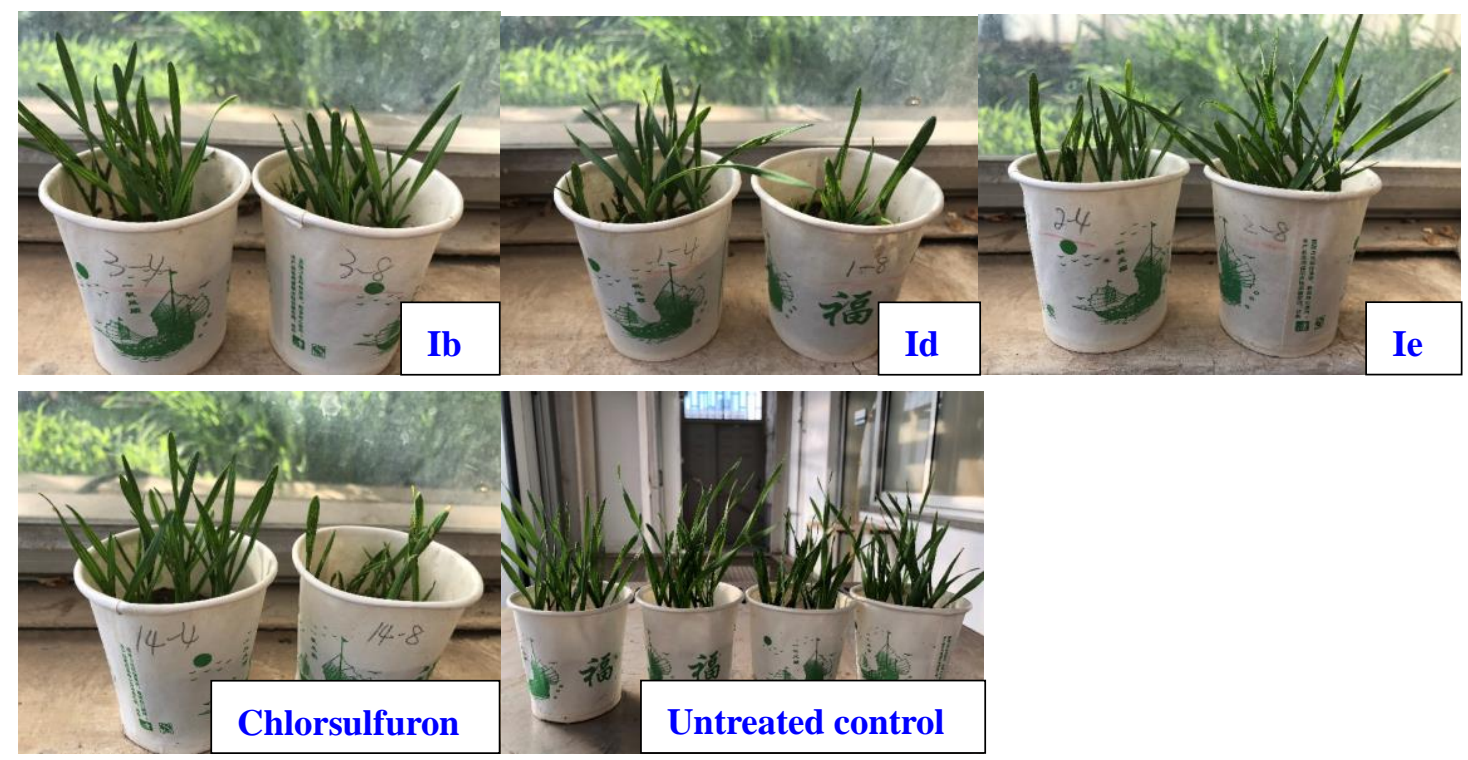

Figure S1 Wheat safety photography under pre-emergence of compounds Ib、Id and Ie. 

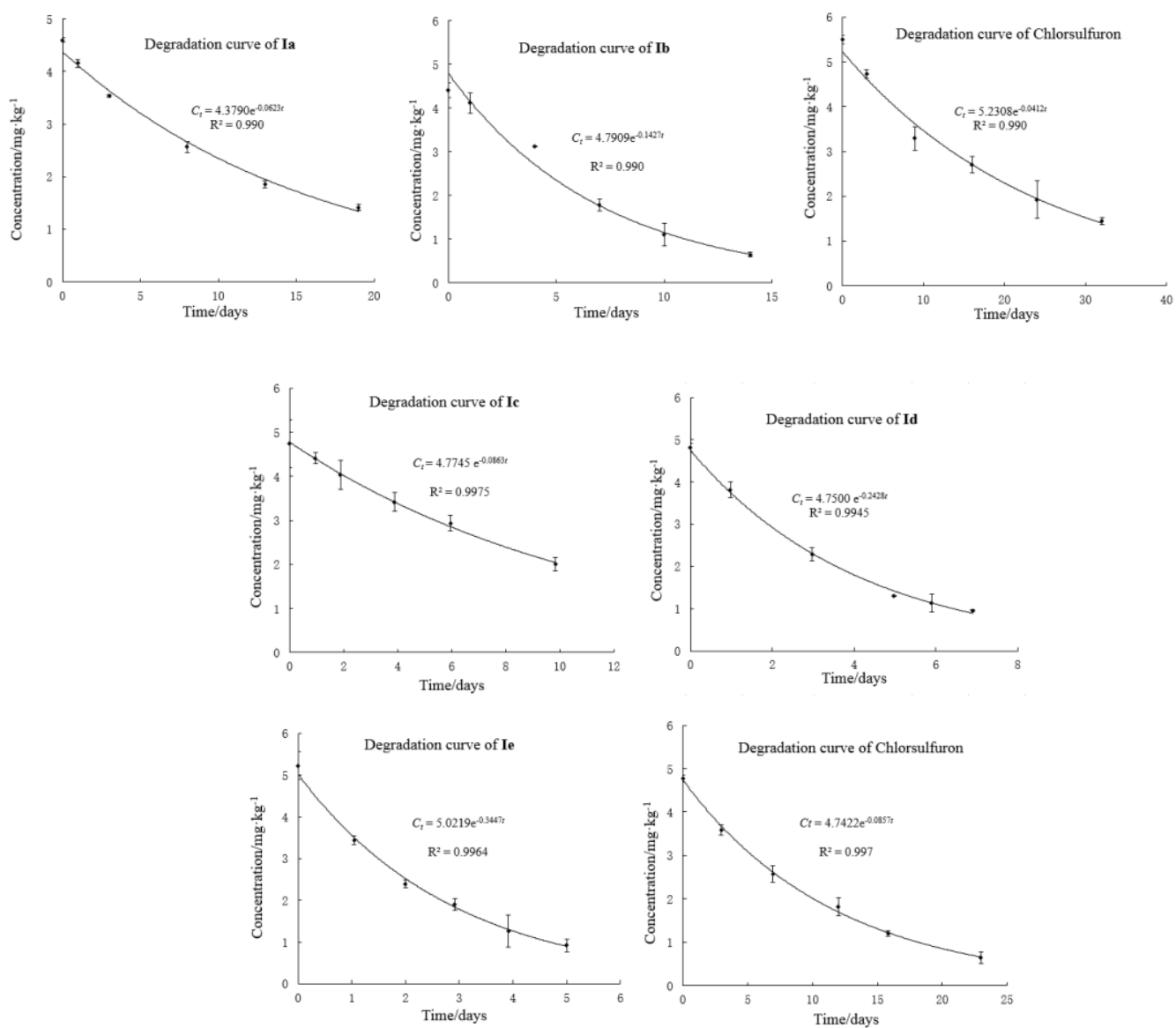

Figure S2 Degradation curves in acidic soil ( $\mathrm{pH} 5.59$ ). 

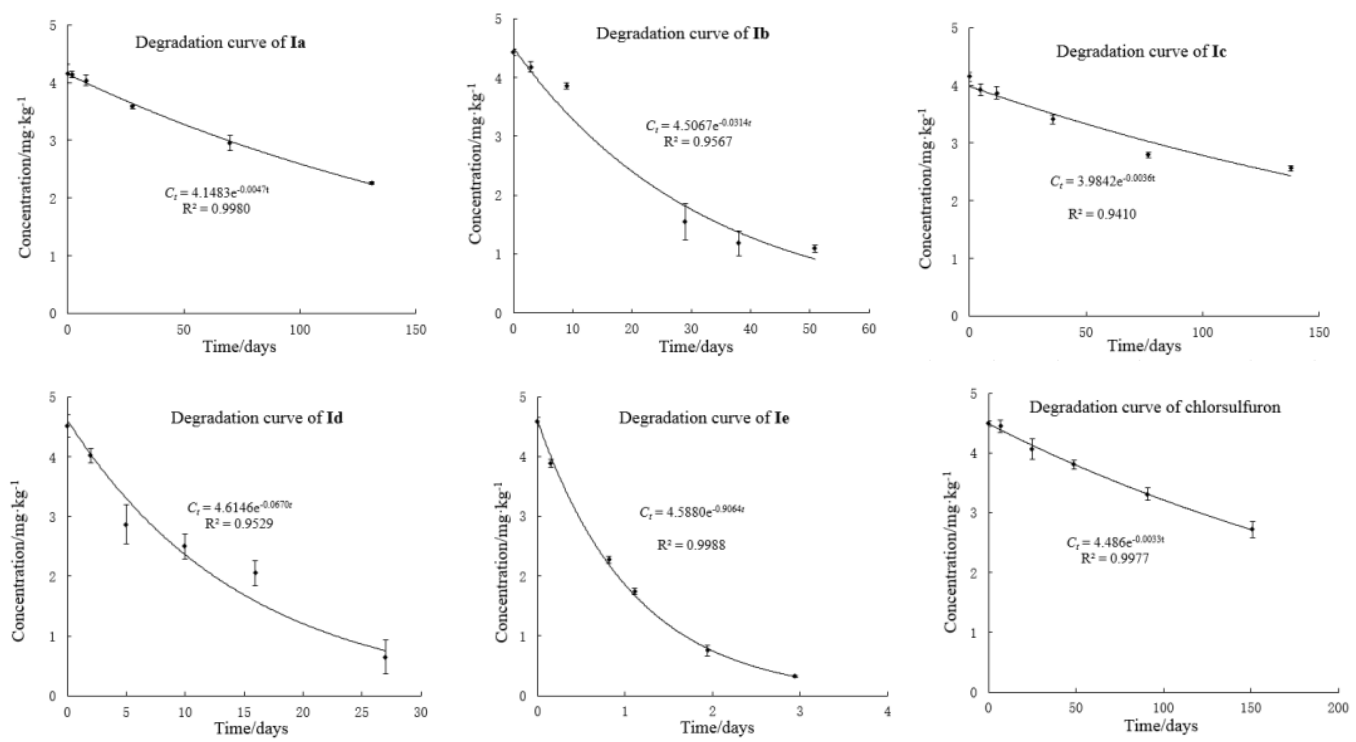

Figure S3 Degradation curves in alkaline soil ( $\mathrm{pH} 8.46)$. 


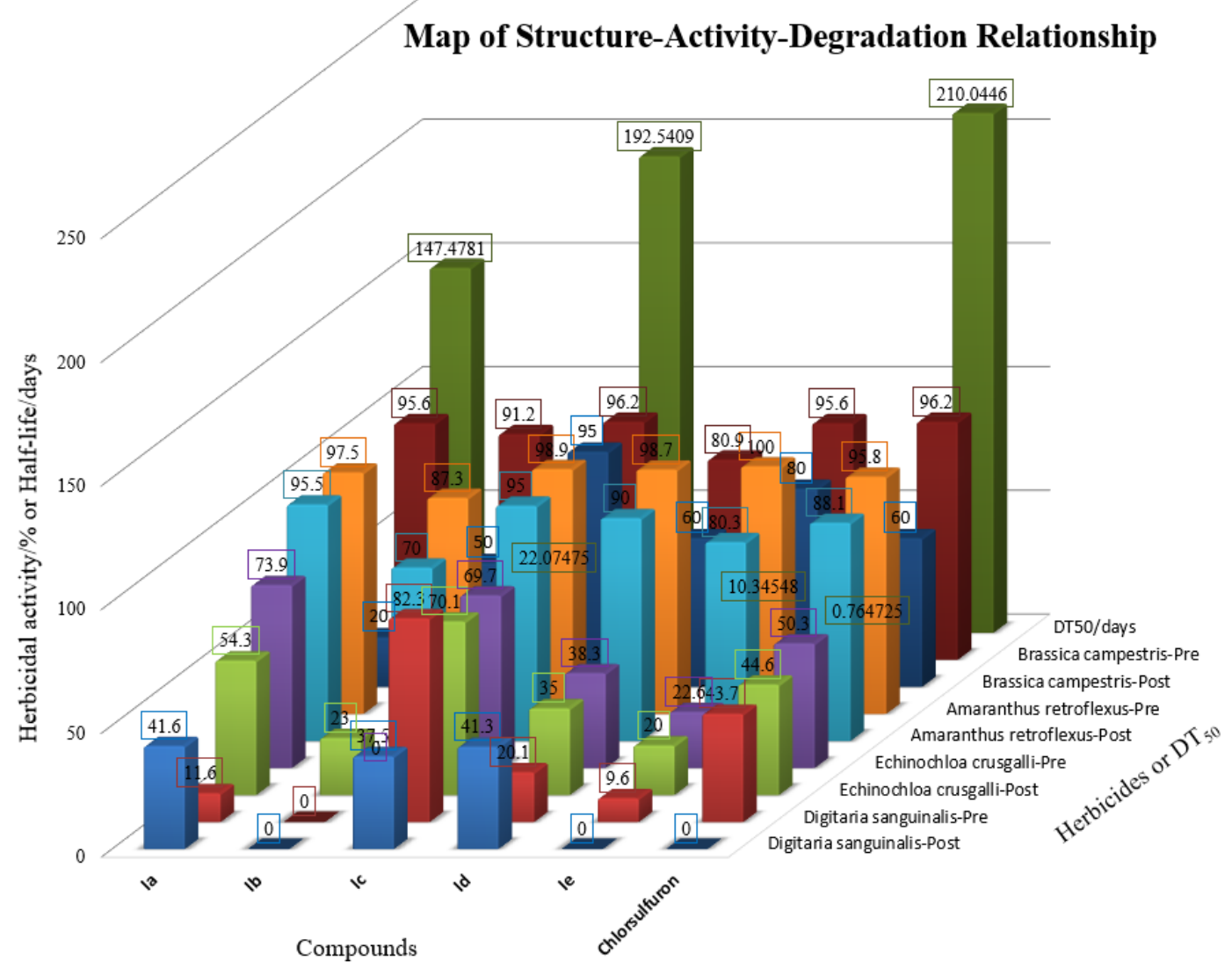

Figure S4 Map of Structure-Activity-Degradation Relationship for target compounds. 
Table S1 Analysis data of Soils

\begin{tabular}{|c|c|c|c|c|c|c|c|c|c|}
\hline \multirow{3}{*}{$\begin{array}{c}\text { Tested } \\
\text { soil }\end{array}$} & \multirow{3}{*}{$\begin{array}{c}\text { Soil } \\
\text { texture }\end{array}$} & \multirow{3}{*}{$\begin{array}{c}\text { Organic } \\
\text { matter } \\
(\%)\end{array}$} & \multirow{3}{*}{$\mathrm{pH}$} & \multirow{3}{*}{$\begin{array}{c}\text { CEC } \\
\left(\mathrm{cmol} \cdot \mathrm{kg}^{-1}\right)\end{array}$} & \multicolumn{5}{|c|}{ Particle size analysis (\%) } \\
\hline & & & & & \multirow{2}{*}{ Sand $^{a}$} & \multicolumn{2}{|c|}{$\operatorname{Silt}^{b}$} & \multicolumn{2}{|c|}{ Clay $^{c}$} \\
\hline & & & & & & Coarse & Fine & Coarse & Fine \\
\hline Acidic & loam & 0.763 & 5.59 & 13.55 & 36 & 10 & 10 & 12 & 32 \\
\hline Alkaline & clay & 0.757 & 8.46 & 10.39 & 30 & 26 & 8 & 8 & 28 \\
\hline
\end{tabular}

${ }^{*}:{ }^{a}$ Sand: $1-0.05 \mathrm{~mm} .{ }^{b}$ Silt: course, $0.05-0.01 \mathrm{~mm}$; fine, $0.01-0.005 \mathrm{~mm} .{ }^{c}$ Clay: course, $0.005-0.001 \mathrm{~mm}$; fine, $<0.001 \mathrm{~mm}$. 
Table S2 HPLC analysis conditions for acidic soil degradation ( $\mathrm{pH}$ 5.59).

\begin{tabular}{|c|c|c|c|}
\hline Compd & $\begin{array}{l}\text { HPLC analysis condition } \\
\text { (wavelength, speed, solvent/v:v) }\end{array}$ & Standard curves & $\begin{array}{l}\text { Coefficient of } \\
\text { correlation }\left(\mathrm{R}^{2}\right)\end{array}$ \\
\hline Ia & $\begin{array}{c}230 \mathrm{~nm}, 0.8 \mathrm{~mL} \cdot \mathrm{min}^{-1} \\
\mathrm{CH}_{3} \mathrm{OH}: \mathrm{H}_{2} \mathrm{O}(\mathrm{pH} 3.0)=70: 30\end{array}$ & $Y=46662.5 X+11656.5$ & 0.9999002 \\
\hline Ib & $\begin{array}{c}230 \mathrm{~nm}, 0.8 \mathrm{~mL} \cdot \mathrm{min}^{-1}, \\
\mathrm{CH}_{3} \mathrm{OH}: \mathrm{H}_{2} \mathrm{O}(\mathrm{pH} 3.0)=70: 30\end{array}$ & $Y=40386.1 X+4512.75$ & 0.9999854 \\
\hline Ic & $\begin{array}{c}230 \mathrm{~nm}, 0.8 \mathrm{~mL} \cdot \mathrm{min}^{-1}, \\
\mathrm{CH}_{3} \mathrm{OH}: \mathrm{H}_{2} \mathrm{O}(\mathrm{pH} 3.0)=70: 30\end{array}$ & $Y=50371.7 X+6065.70$ & 0.9999883 \\
\hline Id & $\begin{array}{c}230 \mathrm{~nm}, 0.8 \mathrm{~mL} \cdot \mathrm{min}^{-1}, \\
\mathrm{CH}_{3} \mathrm{OH}: \mathrm{H}_{2} \mathrm{O}(\mathrm{pH} 3.0)=70: 30\end{array}$ & $Y=34856.8 X+1609.96$ & 0.9999979 \\
\hline Ie & $\begin{array}{c}250 \mathrm{~nm}, 0.8 \mathrm{~mL} \cdot \mathrm{min}^{-1}, \\
\mathrm{CH}_{3} \mathrm{OH}: \mathrm{H}_{2} \mathrm{O}(\mathrm{pH} 3.0)=70: 30\end{array}$ & $\mathrm{Y}=33009.9 \mathrm{X}+3858.21$ & 0.9999861 \\
\hline If & $\begin{array}{c}250 \mathrm{~nm}, 0.8 \mathrm{~mL} \cdot \mathrm{min}^{-1} \\
\mathrm{CH}_{3} \mathrm{OH}: \mathrm{H}_{2} \mathrm{O}(\mathrm{pH} 3.0)=70: 30\end{array}$ & $Y=25743.7 X+1356.74$ & 0.9999902 \\
\hline Ig & $\begin{array}{c}225 \mathrm{~nm}, 0.8 \mathrm{~mL} \cdot \mathrm{min}^{-1}, \\
\mathrm{CH}_{3} \mathrm{OH}: \mathrm{H}_{2} \mathrm{O}(\mathrm{pH} 3.0)=8020\end{array}$ & $Y=52936.9 X+8832.08$ & 0.9999767 \\
\hline Chlorsulfuron & $\begin{array}{c}235 \mathrm{~nm}, 0.8 \mathrm{~mL} \cdot \mathrm{min}^{-1}, \\
\mathrm{CH}_{3} \mathrm{OH}: \mathrm{H}_{2} \mathrm{O}(\mathrm{pH} 3.0)=60: 40\end{array}$ & $\mathrm{Y}=48128.9 \mathrm{X}+11285.2$ & 0.9999212 \\
\hline
\end{tabular}


Table S3 Analytical data for acidic soil degradation ( $\mathrm{pH}$ 5.59).

\begin{tabular}{|c|c|c|c|c|}
\hline Compd & Extraction solvents/v:v & $\begin{array}{c}\text { Adding } \\
\text { concentration } \\
\left(\mathrm{mg} \cdot \mathrm{kg}^{-1}\right)\end{array}$ & $\begin{array}{l}\text { Average } \\
\text { recovery } \\
\text { rate }(\%)\end{array}$ & $\begin{array}{l}\text { Coefficient of } \\
\text { variation }(\%)\end{array}$ \\
\hline \multirow{3}{*}{ Ia } & \multirow{3}{*}{$\begin{array}{c}\mathrm{CH}_{3} \mathrm{COCH}_{3}: \mathrm{CH}_{2} \mathrm{Cl}_{2}: \mathrm{H}_{3} \mathrm{PO}_{4} \text { aqueous } \\
\text { solution }(\mathrm{pH} 2.0)=40: 5: 5\end{array}$} & 5 & 91.30 & 1.54 \\
\hline & & 2 & 84.89 & 2.73 \\
\hline & & 0.5 & 73.02 & 2.40 \\
\hline \multirow{3}{*}{ Ib } & \multirow{3}{*}{$\begin{array}{c}\mathrm{CH}_{3} \mathrm{COCH}_{3}: \mathrm{CH}_{2} \mathrm{Cl}_{2}: \mathrm{H}_{3} \mathrm{PO}_{4} \text { aqueous } \\
\text { solution }(\mathrm{pH} 2.0)=40: 5: 5\end{array}$} & 5 & 91.11 & 1.27 \\
\hline & & 2 & 87.88 & 1.91 \\
\hline & & 0.5 & 83.56 & 1.55 \\
\hline \multirow{3}{*}{ Ic } & \multirow{3}{*}{$\begin{array}{c}\mathrm{CH}_{3} \mathrm{COCH}_{3}: \mathrm{CH}_{2} \mathrm{Cl}_{2}: \mathrm{H}_{3} \mathrm{PO}_{4} \text { aqueous } \\
\text { solution }(\mathrm{pH} 2.0)=40: 5: 5\end{array}$} & 5 & 90.47 & 2.24 \\
\hline & & 2 & 90.11 & 1.54 \\
\hline & & 0.5 & 86.11 & 2.05 \\
\hline \multirow{3}{*}{ Id } & $\mathrm{CH}_{3} \mathrm{COCH}_{3}: \mathrm{CH}_{2} \mathrm{Cl}_{2}: \mathrm{THF}: \mathrm{H}_{3} \mathrm{PO}_{4}$ & 5 & 84.37 & 2.10 \\
\hline & aqueous solution $(\mathrm{pH} 2.0)=$ & 2 & 79.63 & 1.91 \\
\hline & $30: 5: 5: 5$ & 0.5 & 74.32 & 2.49 \\
\hline \multirow{3}{*}{ Ie } & \multirow{3}{*}{$\begin{array}{c}\mathrm{CH}_{3} \mathrm{COCH}_{3}: \mathrm{CH}_{2} \mathrm{Cl}_{2}: \mathrm{H}_{3} \mathrm{PO}_{4} \text { aqueous } \\
\text { solution }(\mathrm{pH} 2.0)=40: 5: 5\end{array}$} & 5 & 89.12 & 1.15 \\
\hline & & 2 & 87.85 & 0.85 \\
\hline & & 0.5 & 82.48 & 1.73 \\
\hline \multirow{3}{*}{ Chlorsulfuron } & \multirow{3}{*}{$\begin{array}{c}\mathrm{CH}_{3} \mathrm{COCH}_{3}: \mathrm{CH}_{2} \mathrm{Cl}_{2}: \mathrm{H}_{3} \mathrm{PO}_{4} \text { aqueous } \\
\text { solution }(\mathrm{pH} 2.0)=40: 5: 5\end{array}$} & 5 & 87.96 & 1.91 \\
\hline & & 2 & 85.69 & 1.92 \\
\hline & & 0.5 & 75.46 & 3.53 \\
\hline
\end{tabular}


Table S4 HPLC analysis conditions for alkaline soil degradation ( $\mathrm{pH} 8.46$ ).

\begin{tabular}{|c|c|c|c|}
\hline Compd & $\begin{array}{c}\text { HPLC analysis condition } \\
\text { (wavelength, speed, solvent/v:v) }\end{array}$ & Standard curves & $\begin{array}{l}\text { Coefficient of } \\
\text { correlation }\left(\mathrm{R}^{2}\right)\end{array}$ \\
\hline Ia & $\begin{array}{c}230 \mathrm{~nm}, 0.5 \mathrm{~mL} \cdot \mathrm{min}^{-1}, \\
\mathrm{CH}_{3} \mathrm{OH}: \mathrm{H}_{2} \mathrm{O}(\mathrm{pH} 3.0)=70: 30\end{array}$ & $Y=43195.5 X+1301.15$ & 0.9999611 \\
\hline Ib & $\begin{array}{c}230 \mathrm{~nm}, 0.8 \mathrm{~mL} \cdot \mathrm{min}^{-1}, \\
\mathrm{CH}_{3} \mathrm{OH}: \mathrm{H}_{2} \mathrm{O}(\mathrm{pH} 3.0)=75: 25\end{array}$ & $Y=42389.5 X+11772.9$ & 0.9999084 \\
\hline Ic & $\begin{array}{c}230 \mathrm{~nm}, 0.8 \mathrm{~mL} \cdot \mathrm{min}^{-1}, \\
\mathrm{CH}_{3} \mathrm{OH}: \mathrm{H}_{2} \mathrm{O}(\mathrm{pH} 3.0)=70: 30\end{array}$ & $Y=52793.5 X+11357.4$ & 0.9999210 \\
\hline Id & $\begin{array}{c}230 \mathrm{~nm}, 0.8 \mathrm{~mL} \cdot \mathrm{min}^{-1}, \\
\mathrm{CH}_{3} \mathrm{OH}: \mathrm{H}_{2} \mathrm{O}(\mathrm{pH} 3.0)=70: 30\end{array}$ & $Y=35846.4 X+4186.64$ & 0.9999521 \\
\hline Ie & $\begin{array}{c}250 \mathrm{~nm}, 0.8 \mathrm{~mL} \cdot \mathrm{min}^{-1} \\
\mathrm{CH}_{3} \mathrm{OH}: \mathrm{H}_{2} \mathrm{O}(\mathrm{pH} 3.0)=70: 30\end{array}$ & $Y=32347.7 X+72.9110$ & 0.9999974 \\
\hline If & $\begin{array}{c}250 \mathrm{~nm}, 0.8 \mathrm{~mL} \cdot \mathrm{min}^{-1}, \\
\mathrm{CH}_{3} \mathrm{OH}: \mathrm{H}_{2} \mathrm{O}(\mathrm{pH} 3.0)=70: 30\end{array}$ & $Y=28664.0 X+1119.31$ & 0.9999970 \\
\hline Ig & $\begin{array}{c}225 \mathrm{~nm}, 0.8 \mathrm{~mL} \cdot \mathrm{min}^{-1} \\
\mathrm{CH}_{3} \mathrm{OH}: \mathrm{H}_{2} \mathrm{O}(\mathrm{pH} 3.0)=8020\end{array}$ & $Y=56030.6 X+5443.73$ & 0.9999939 \\
\hline Chlorsulfuron & $\begin{array}{c}235 \mathrm{~nm}, 0.8 \mathrm{~mL} \cdot \mathrm{min}^{-1}, \\
\mathrm{CH}_{3} \mathrm{OH}: \mathrm{H}_{2} \mathrm{O}(\mathrm{pH} 3.0)=60: 40\end{array}$ & $Y=40709.1 X+2929.16$ & 0.9999823 \\
\hline
\end{tabular}


Table S5 Analytical data for alkaline soil degradation ( $\mathrm{pH}$ 8.46).

\begin{tabular}{|c|c|c|c|c|}
\hline Compd & Extraction solvents/v:v & $\begin{array}{l}\text { Adding } \\
\text { concentration } \\
\left(\mathrm{mg} \cdot \mathrm{kg}^{-1}\right)\end{array}$ & $\begin{array}{l}\text { Average } \\
\text { recovery rate } \\
(\%)\end{array}$ & $\begin{array}{l}\text { Coefficient of } \\
\text { variation }(\%)\end{array}$ \\
\hline \multirow{3}{*}{ Ia } & \multirow{3}{*}{$\begin{array}{c}\qquad \mathrm{CH}_{3} \mathrm{COCH}_{3}: \mathrm{CH}_{2} \mathrm{Cl}_{2}: \mathrm{H}_{3} \mathrm{PO}_{4} \\
\text { aqueous solution }(\mathrm{pH} 1.5)=40: 5: 5\end{array}$} & 5 & 87.86 & 1.74 \\
\hline & & 2 & 82.96 & 1.31 \\
\hline & & 0.5 & 72.80 & 0.83 \\
\hline \multirow{3}{*}{ Ib } & \multirow{3}{*}{$\begin{array}{c}\mathrm{CH}_{3} \mathrm{COCH}_{3}: \mathrm{CH}_{2} \mathrm{Cl}_{2}: \mathrm{H}_{3} \mathrm{PO}_{4} \\
\text { aqueous solution }(\mathrm{pH} 1.5)=40: 5: 5\end{array}$} & 5 & 92.15 & 1.24 \\
\hline & & 2 & 86.09 & 2.07 \\
\hline & & 0.5 & 72.98 & 2.25 \\
\hline \multirow{3}{*}{ Ic } & \multirow{3}{*}{$\begin{array}{c}\qquad \mathrm{CH}_{3} \mathrm{COCH}_{3}: \mathrm{CH}_{2} \mathrm{Cl}_{2}: \mathrm{H}_{3} \mathrm{PO}_{4} \\
\text { aqueous solution }(\mathrm{pH} 1.5)=40: 5: 5\end{array}$} & 5 & 84.73 & 3.77 \\
\hline & & 2 & 72.38 & 2.11 \\
\hline & & 0.5 & 73.73 & 3.83 \\
\hline \multirow{3}{*}{ Id } & \multirow{3}{*}{$\begin{array}{c}\mathrm{CH}_{3} \mathrm{COCH}_{3}: \mathrm{CH}_{2} \mathrm{Cl}_{2}: \mathrm{H}_{3} \mathrm{PO}_{4} \\
\text { aqueous solution }(\mathrm{pH} 1.5)=40: 5: 5\end{array}$} & 5 & 83.34 & 2.01 \\
\hline & & 2 & 77.74 & 3.03 \\
\hline & & 0.5 & 73.40 & 0.26 \\
\hline \multirow{3}{*}{ Ie } & \multirow{3}{*}{$\begin{array}{c}\mathrm{CH}_{3} \mathrm{COCH}_{3}: \mathrm{CH}_{2} \mathrm{Cl}_{2}: \mathrm{H}_{3} \mathrm{PO}_{4} \\
\text { aqueous solution }(\mathrm{pH} 1.5)=40: 5: 5\end{array}$} & 5 & 85.62 & 3.38 \\
\hline & & 2 & 80.98 & 2.23 \\
\hline & & 0.5 & 85.38 & 1.88 \\
\hline \multirow{3}{*}{ Chlorsulfuron } & $\mathrm{CH}_{3} \mathrm{COCH}_{3}: \mathrm{CH}_{2} \mathrm{Cl}_{2}: \mathrm{H}_{3} \mathrm{PO}_{4}$ & 5 & 88.58 & 2.15 \\
\hline & aqueous solution $(\mathrm{pH} 1.5)=$ & 2 & 84.55 & 2.84 \\
\hline & $40: 5: 10$ & 0.5 & 70.24 & 3.95 \\
\hline
\end{tabular}


Table S6 Root inhibition of oilseed rape (B. campestris).

\begin{tabular}{|c|c|c|c|c|c|}
\hline \multirow{2}{*}{ Compd } & \multirow{2}{*}{$\begin{array}{c}\text { Concn } \\
\left(\mu \mathrm{g} \cdot \mathrm{mL}^{-1}\right)\end{array}$} & \multirow{2}{*}{$\begin{array}{l}\text { Length of hypocotyl } \\
\qquad(\mathrm{mm})\end{array}$} & \multicolumn{2}{|c|}{ Variance analysis ${ }^{\mathrm{a}}$} & \multirow{2}{*}{ Inhibition rate $(\%)$} \\
\hline & & & $5 \%$ & $1 \%$ & \\
\hline \multirow[t]{2}{*}{$\mathrm{ck}$} & 0 & 42.96 & $a b$ & A & - \\
\hline & 10 & 12.00 & ghij & FGH & 72.1 \\
\hline \multirow[t]{3}{*}{ Ia } & 1 & 13.38 & fghij & DEFGH & 68.9 \\
\hline & 0.1 & 17.69 & de & $\mathrm{CDEF}$ & 58.8 \\
\hline & 10 & 11.00 & ghij & $\mathrm{H}$ & 74.4 \\
\hline \multirow[t]{3}{*}{ Ib } & 1 & 13.19 & fghij & DEFGH & 69.3 \\
\hline & 0.1 & 25.88 & $\mathrm{c}$ & B & 39.8 \\
\hline & 10 & 11.18 & ghij & $\mathrm{H}$ & 74.0 \\
\hline \multirow[t]{3}{*}{ Ic } & 1 & 13.94 & efghij & DEFGH & 67.6 \\
\hline & 0.1 & 25.13 & $\mathrm{c}$ & B & 41.5 \\
\hline & 10 & 11.85 & ghij & FGH & 72.4 \\
\hline \multirow[t]{2}{*}{ Id } & 1 & 12.19 & ghij & EFGH & 71.6 \\
\hline & 0.1 & 12.50 & ghij & DEFGH & 70.9 \\
\hline \multirow{3}{*}{ Ie } & 10 & 11.13 & ghij & $\mathrm{H}$ & 74.1 \\
\hline & 1 & 12.44 & ghij & DEFGH & 71.0 \\
\hline & 0.1 & 28.88 & $\mathrm{c}$ & B & 32.8 \\
\hline \multirow{3}{*}{ If } & 10 & 10.31 & hij & $\mathrm{H}$ & 76.0 \\
\hline & 1 & 17.13 & def & CDEFG & 60.1 \\
\hline & 0.1 & 39.75 & $\mathrm{~b}$ & A & 7.5 \\
\hline \multirow{3}{*}{ Ig } & 10 & 12.41 & ghij & DEFGH & 71.1 \\
\hline & 1 & 15.44 & efg & CDEFGH & 64.1 \\
\hline & 0.1 & 25.75 & $\mathrm{c}$ & B & 40.1 \\
\hline \multirow{3}{*}{ Chlorsulfuron } & 10 & 10.06 & $\mathrm{ij}$ & $\mathrm{H}$ & 76.6 \\
\hline & 1 & 13.31 & fghij & DEFGH & 69.0 \\
\hline & 0.1 & 14.88 & efgh & CDEFGH & 65.4 \\
\hline
\end{tabular}

${ }^{*}:{ }^{a}$ Different letters within the same column indicate significant difference at $5 \%$ or $1 \%$ level 
Table S7 Wheat safety of compound Ic

\begin{tabular}{|c|c|c|c|c|c|c|c|c|c|}
\hline \multirow{4}{*}{ Compd } & \multirow{4}{*}{$\begin{array}{c}\text { Concn } \\
\left(\text { a.i } \mathrm{g} \cdot \mathrm{ha}^{-1}\right)\end{array}$} & \multicolumn{8}{|c|}{ Wheat (Jimai 22) } \\
\hline & & \multicolumn{4}{|c|}{ Pre (22 days) } & \multicolumn{4}{|c|}{ Post(28 days) } \\
\hline & & \multirow{2}{*}{$\begin{array}{l}\text { Fresh } \\
\text { weight } \\
\text { (g/cup) }\end{array}$} & \multicolumn{2}{|c|}{ Variance analysis ${ }^{a}$} & \multirow{2}{*}{$\begin{array}{l}\text { Contrast } \\
\text { ratio } \%^{\mathrm{b}}\end{array}$} & \multirow{2}{*}{$\begin{array}{l}\text { Fresh } \\
\text { weight } \\
\text { (g/cup } \\
\text { ) }\end{array}$} & \multicolumn{2}{|c|}{ Variance analysis ${ }^{a}$} & \multirow{2}{*}{$\begin{array}{l}\text { Contras } \\
\text { ratio } \%\end{array}$} \\
\hline & & & $5 \%$ & $1 \%$ & & & $5 \%$ & $1 \%$ & \\
\hline & 0 & 2.526 & $\mathrm{ab}$ & $\mathrm{AB}$ & - & 2.514 & $a b$ & $\mathrm{ABC}$ & - \\
\hline \multirow{5}{*}{ Ic } & 15 & 1.400 & cdefghijk & BCDEF & -44.6 & 2.413 & $a b$ & $\mathrm{ABC}$ & -4.1 \\
\hline & & & & G & & & & & \\
\hline & 30 & 0.919 & fghijklm & EFGHI & -63.6 & 2.369 & $a b c$ & $\mathrm{ABC}$ & -5.8 \\
\hline & 60 & 0.588 & ijklm & FGHI & -76.7 & 2.270 & abcd & $\mathrm{ABC}$ & -9.7 \\
\hline & 120 & 0.333 & $\operatorname{lm}$ & GHI & -86.8 & 2.268 & abcd & $\mathrm{ABC}$ & -9.8 \\
\hline \multirow{4}{*}{$\begin{array}{l}\text { Chlorsul- } \\
\text { furon }\end{array}$} & 15 & 2.529 & $a b$ & $\mathrm{AB}$ & 0.1 & 2.570 & $a b$ & $\mathrm{AB}$ & 2.2 \\
\hline & 30 & 3.171 & $\mathrm{a}$ & A & 25.6 & 2.495 & $a b$ & $\mathrm{ABC}$ & -0.8 \\
\hline & 60 & 2.292 & $\mathrm{bc}$ & $\mathrm{ABCD}$ & -9.3 & 2.320 & $a b c$ & $\mathrm{ABC}$ & -7.7 \\
\hline & 120 & 2.231 & bcd & $\mathrm{ABCD}$ & -11.7 & 2.085 & abcd & $\mathrm{ABC}$ & -17.1 \\
\hline
\end{tabular}

${ }^{*}:{ }^{a}$ Different letters within the same column indicate significant difference at $5 \%$ or $1 \%$ level; ${ }^{b}$ Contrast ratio $\%$, negative means 
Table S8 Corn safety of compound Ic

\begin{tabular}{|c|c|c|c|c|c|}
\hline \multirow{4}{*}{ Compd } & \multirow{4}{*}{$\begin{array}{c}\text { Concn } \\
\left(\text { a.i } \mathrm{g} \cdot \mathrm{ha}^{-1}\right)\end{array}$} & \multicolumn{4}{|c|}{ Corn (Jinbei 288) } \\
\hline & & \multicolumn{4}{|c|}{ Pre-emergence (22 days) } \\
\hline & & \multirow{2}{*}{$\begin{array}{c}\text { Fresh weight } \\
\text { (g/5 plants) }\end{array}$} & \multicolumn{2}{|c|}{ Variance analysis ${ }^{a}$} & \multirow{2}{*}{$\begin{array}{l}\text { Contrast } \\
\text { ratio } \%^{\mathrm{b}}\end{array}$} \\
\hline & & & $5 \%$ & $1 \%$ & \\
\hline & 0 & 7.990 & $\mathrm{a}$ & A & - \\
\hline \multirow{3}{*}{ Ic } & 15 & 5.567 & $\mathrm{bc}$ & $\mathrm{ABCD}$ & -30.3 \\
\hline & 30 & 5.024 & bcd & $\mathrm{ABCD}$ & -37.1 \\
\hline & 60 & 3.553 & cde & DEF & -55.5 \\
\hline \multirow{3}{*}{ Chlorsulfuron } & 30 & 0.559 & fg & FG & -93.0 \\
\hline & 60 & 0.060 & $\mathrm{~g}$ & G & -99.2 \\
\hline & 120 & 0.063 & $\mathrm{~g}$ & $\mathrm{G}$ & -99.2 \\
\hline
\end{tabular}

${ }^{*}:{ }^{a}$ Different letters within the same column indicate significant difference at $5 \%$ or $1 \%$ level; ${ }^{b}$ Contrast ratio $\%$, negative means inhibition, positive means promotion. 
Table S9 Wheat safety of compounds Ib、Id and Ie.

\begin{tabular}{|c|c|c|c|c|c|}
\hline \multirow{3}{*}{ Compd } & \multirow{3}{*}{$\begin{array}{c}\text { Concn } \\
\left(\text { a.i g.ha }{ }^{-1}\right)\end{array}$} & \multirow{3}{*}{$\begin{array}{l}\text { Fresh weight } \\
\text { (g/ cup) }\end{array}$} & \multicolumn{3}{|c|}{ Wheat (Jimai 22) } \\
\hline & & & \multicolumn{2}{|c|}{ Variance analysis ${ }^{a}$} & \multirow{2}{*}{$\begin{array}{l}\text { Contrast } \\
\text { ratio } \%^{\mathrm{b}}\end{array}$} \\
\hline & & & $5 \%$ & $1 \%$ & \\
\hline & 0 & 1.175 & $a b c$ & $\mathrm{ABC}$ & - \\
\hline \multirow{2}{*}{ Ib } & 60 & 1.007 & abcde & $\mathrm{ABCDE}$ & -14.3 \\
\hline & 120 & 0.837 & cdefg & BCDEFG & -28.8 \\
\hline \multirow{2}{*}{ Id } & 60 & 0.753 & efghij & CDEFGH & -35.9 \\
\hline & 120 & 0.500 & ghijkl & FGH & -57.4 \\
\hline \multirow{2}{*}{ Ie } & 60 & 1.023 & abcde & ABCDE & -12.9 \\
\hline & 120 & 1.033 & abcde & $\mathrm{ABCDE}$ & -12.1 \\
\hline \multirow{2}{*}{ Chlorsulfuron } & 60 & 0.957 & bcdef & ABCDEF & -18.6 \\
\hline & 120 & 0.717 & efghij & CDEFGH & -39.0 \\
\hline
\end{tabular}

${ }^{*}:{ }^{a}$ Different letters within the same column indicate significant difference at $5 \%$ or $1 \%$ level; ${ }^{b}$ Contrast ratio $\%$, negative means inhibition, positive means promotion. 


\section{Data 2}

Ethyl

(4-chloro-3-(N-((4-methoxy-6-methyl-1,3,5-triazin-2-yl)carbamoyl)sulfamoyl)phenyl)

(methyl)carbamate Ia. Light yellow solid, yield 88\%, m.p. 86-88 ${ }^{\circ}$ C. ${ }^{1} \mathrm{H}$ NMR (400 MHz, DMSO) $\delta 12.89$ (s, 1H, NH), 11.09 (s, 1H, NH), 8.10 (s, 1H, Ph-H), 7.70 (s, 2H, $\mathrm{Ph}-\mathrm{H}), 4.13\left(\mathrm{q}, J=14.1,7.0 \mathrm{~Hz}, 2 \mathrm{H}, \mathrm{OCH}_{2} \mathrm{CH}_{3}\right), 3.97\left(\mathrm{~s}, 3 \mathrm{H}, \mathrm{NCH}_{3}\right), 3.28(\mathrm{~s}, 3 \mathrm{H}$, $\left.\mathrm{OCH}_{3}\right), 2.46\left(\mathrm{~s}, 3 \mathrm{H}, \mathrm{CH}_{3}\right), 1.21\left(\mathrm{t}, J=7.1 \mathrm{~Hz}, 3 \mathrm{H}, \mathrm{OCH}_{2} \mathrm{CH}_{3}\right)$. HRMS (ESI) Calcd for $\mathrm{C}_{16} \mathrm{H}_{19} \mathrm{ClN}_{6} \mathrm{O}_{6} \mathrm{~S}[\mathrm{M}+\mathrm{H}]^{+} 459.0775$, found 459.0849.

Tert-butyl

(4-chloro-3-(N-((4-methoxy-6-methyl-1,3,5-triazin-2-yl)carbamoyl)sulfamoyl)phenyl) (methyl)carbamate Ib. White solid, yield 81\%, m.p. 147-149 ${ }^{\circ} \mathrm{C} .{ }^{1} \mathrm{H}$ NMR (400 MHz, DMSO) $\delta 12.90(\mathrm{~s}, 1 \mathrm{H}, \mathrm{NH}), 11.09(\mathrm{~s}, 1 \mathrm{H}, \mathrm{NH}), 8.08(\mathrm{~s}, 1 \mathrm{H}, \mathrm{Ph}-\mathrm{H}), 7.67(\mathrm{~s}, 2 \mathrm{H}$, Ph-H), $3.97\left(\mathrm{~s}, 3 \mathrm{H}, \mathrm{NCH}_{3}\right), 3.24\left(\mathrm{~s}, 3 \mathrm{H}, \mathrm{OCH}_{3}\right), 2.46\left(\mathrm{~s}, 3 \mathrm{H}, \mathrm{CH}_{3}\right), 1.44(\mathrm{~s}, 9 \mathrm{H}$, $\left.\mathrm{C}\left(\mathrm{CH}_{3}\right)_{3}\right)$. HRMS (ESI) Calcd for $\mathrm{C}_{18} \mathrm{H}_{23} \mathrm{ClN}_{6} \mathrm{O}_{6} \mathrm{~S}[\mathrm{M}+\mathrm{H}]^{+}$487.1088, found 487.1158. $N$-(4-Chloro-3-(N-((4-methoxy-6-methyl-1,3,5-triazin-2-yl)carbamoyl)sulfamoyl)phe nyl)-2,2,2-trifluoro- $N$-methylacetamide Ic. White solid, yield $85 \%$, m.p. $133-135{ }^{\circ} \mathrm{C}$. ${ }^{1} \mathrm{H}$ NMR (400 MHz, DMSO) $\delta 12.90(\mathrm{~s}, 1 \mathrm{H}, \mathrm{NH}), 11.07$ (s, 1H, NH), $8.10(\mathrm{~s}, 1 \mathrm{H}$, Ph-H), 7.70 (s, 2H, Ph-H), 3.98 (s, 3H, NCH$), 3.28\left(\mathrm{~s}, 3 \mathrm{H}, \mathrm{OCH}_{3}\right), 2.46$ (s, 3H, $\left.\mathrm{CH}_{3}\right)$. HRMS (ESI) Calcd for $\mathrm{C}_{15} \mathrm{H}_{14} \mathrm{ClF}_{3} \mathrm{~N}_{6} \mathrm{O}_{5} \mathrm{~S}[\mathrm{M}+\mathrm{H}]^{+} 483.0387$, found 483.0457. 
2-Chloro- $N$-((4-methoxy-6-methyl-1,3,5-triazin-2-yl)carbamoyl)-5-(methylamino)ben zenesulfonamide Id. White solid, yield 80\%, m.p. 157-159 ${ }^{\circ} \mathrm{C} .{ }^{1} \mathrm{H}$ NMR (400 MHz, DMSO) $\delta 13.01(\mathrm{~s}, 1 \mathrm{H}, \mathrm{NH}), 11.07$ (s, 1H, NH), 8.27 (s, 1H, Ph-H), 7.87 (s, 2H, Ph-H, $\left.\mathrm{CH}_{3}-\mathrm{NH}\right), 7.45(\mathrm{~d}, J=119.5 \mathrm{~Hz}, 1 \mathrm{H}, \mathrm{Ph}-\mathrm{H}), 3.97\left(\mathrm{~s}, 3 \mathrm{H}, \mathrm{OCH}_{3}\right), 3.30(\mathrm{~s}, 3 \mathrm{H}$, $\mathrm{CH}_{3}-\mathrm{NH}$ ), 2.46 (s, 3H, $\mathrm{CH}_{3}$ ). HRMS (ESI) Calcd for $\mathrm{C}_{13} \mathrm{H}_{15} \mathrm{ClN}_{6} \mathrm{O}_{4} \mathrm{~S}[\mathrm{M}+\mathrm{H}]^{+}$ 387.0564, found 387.0631 .

$N$-(4-Chloro-3-(N-((4-methoxy-6-methyl-1,3,5-triazin-2-yl)carbamoyl)sulfamoyl)phe nyl)-2,2,2-trifluoroacetamide Ie. White solid, yield 85\%, m.p. 201-203 ${ }^{\circ} \mathrm{C} .{ }^{1} \mathrm{H}$ NMR (400 MHz, DMSO) $\delta 12.94(\mathrm{~s}, 1 \mathrm{H}, \mathrm{NH}), 11.77$ (s, 1H, TFA-NH), $11.11(\mathrm{~s}, 1 \mathrm{H}, \mathrm{NH})$, $8.55(\mathrm{~s}, 1 \mathrm{H}, \mathrm{Ph}-\mathrm{H}), 8.11(\mathrm{~d}, J=8.8 \mathrm{~Hz}, 1 \mathrm{H}, \mathrm{Ph}-\mathrm{H}), 7.78(\mathrm{~d}, J=8.6 \mathrm{~Hz}, 1 \mathrm{H}, \mathrm{Ph}-\mathrm{H})$, $3.98\left(\mathrm{~s}, 3 \mathrm{H}, \mathrm{OCH}_{3}\right), 2.47\left(\mathrm{~s}, 3 \mathrm{H}, \mathrm{CH}_{3}\right)$. HRMS (ESI) Calcd for $\mathrm{C}_{14} \mathrm{H}_{12} \mathrm{ClF}_{3} \mathrm{~N}_{6} \mathrm{O}_{5} \mathrm{~S}$ $[\mathrm{M}+\mathrm{H}]^{+}$469.0231, found 469.0299.

2-chloro- $N$-((4-methoxy-6-methyl-1,3,5-triazin-2-yl)carbamoyl)-5-(1H-pyrrol)benzen esulfonamide If. White solid, yield 85\%, m.p. $186-188{ }^{\circ} \mathrm{C} .{ }^{1} \mathrm{H}$ NMR (400 MHz, Acetone) $\delta(\mathrm{ppm}) 13.07(\mathrm{~s}, 1 \mathrm{H}, \mathrm{NH}), 9.86(\mathrm{~s}, 1 \mathrm{H}, \mathrm{NH}), 8.24(\mathrm{~d}, J=2.2 \mathrm{~Hz}, 1 \mathrm{H}, \mathrm{Ph}-\mathrm{H})$, $7.89(\mathrm{dd}, J=8.5,2.2 \mathrm{~Hz}, 1 \mathrm{H}, \mathrm{Ph}-\mathrm{H}), 7.72(\mathrm{~d}, J=8.6 \mathrm{~Hz}, 1 \mathrm{H}, \mathrm{Ph}-\mathrm{H}), 7.37(\mathrm{~s}, 2 \mathrm{H}$, -CH-CH-), 6.34 (s, 2H, -CH-CH-), 4.01 (s, 3H, $\left.\mathrm{OCH}_{3}\right), 2.02$ (s, 3H, $\left.\mathrm{CH}_{3}\right)$. Anal. Calc. for $\mathrm{C}_{16} \mathrm{H}_{15} \mathrm{ClN}_{6} \mathrm{O}_{4} \mathrm{~S}$ : C 45.45, H 3.58, N 19.88. Found: C 45.29, H 3.43, N 20.11. 
amoyl)benzenesulfonamide Ig. White solid, yield 84\%, m.p. 191-193 ${ }^{\circ} \mathrm{C} .{ }^{1} \mathrm{H}$ NMR (400 MHz, DMSO) $\delta(\mathrm{ppm}) 13.01(\mathrm{~s}, 1 \mathrm{H}, \mathrm{NH}), 11.14(\mathrm{~s}, 1 \mathrm{H}, \mathrm{NH}), 7.87(\mathrm{~d}, J=8.1 \mathrm{~Hz}$ 2H, Ph-H), 7.74 (dd, J=8.4, 1.9 Hz, 1H, Ph-H), 5.87 (s, 2H, -CH-CH-), 3.98 (s, 3H, $\left.\mathrm{OCH}_{3}\right), 2.47\left(\mathrm{~s}, 3 \mathrm{H}, \mathrm{CH}_{3}\right), 2.01\left(\mathrm{~s}, 6 \mathrm{H},\left(\mathrm{CH}_{3}\right)_{2}\right)$. Anal. Calc. for $\mathrm{C}_{18} \mathrm{H}_{19} \mathrm{ClN}_{6} \mathrm{O}_{4} \mathrm{~S}: \mathrm{C}$ 47.95, H 4.25, N 18.64. Found: C 47.81, H 4.32, N 18.43.

$N$-(4-Chloro-3-sulfamoylphenyl)-2,2,2-trifluoroacetamide 4. ${ }^{1} \mathrm{H}$ NMR (400 MHz, DMSO) $\delta 11.64(\mathrm{~s}, 1 \mathrm{H}, \mathrm{NH}), 8.40(\mathrm{~d}, J=2.5 \mathrm{~Hz}, 1 \mathrm{H}, \mathrm{Ph}-\mathrm{H}), 7.91(\mathrm{dd}, J=8.7,2.6 \mathrm{~Hz}$, 1H, Ph-H), $7.75-7.66\left(\mathrm{~m}, 3 \mathrm{H}, \mathrm{NH}_{2}, \mathrm{Ph}-\mathrm{H}\right)$.

$N$-(4-Chloro-3-(N-((dimethylamino)methylene)sulfamoyl)phenyl)-2,2,2-trifluoroaceta mide 5. ${ }^{1} \mathrm{H}$ NMR (400 MHz, DMSO) $\delta 11.62(\mathrm{~s}, 1 \mathrm{H}, \mathrm{NH}), 8.44(\mathrm{~s}, 1 \mathrm{H}, \mathrm{CH}=\mathrm{N}), 8.28$ (s, 1H, Ph-H), 7.90 (s, 1H, Ph-H), 7.64 (s, 1H, Ph-H), 3.20 (s, 3H, $\left.\mathrm{CH}_{3}\right), 2.94$ (s, 3H, $\left.\mathrm{CH}_{3}\right)$.

$N$-(4-Chloro-3-(N-((dimethylamino)methylene)sulfamoyl)phenyl)-2,2,2-trifluoro- $N$-m ethylacetamide 6. ${ }^{1} \mathrm{H}$ NMR (400 MHz, DMSO) $\delta 8.35(\mathrm{~s}, 1 \mathrm{H}, \mathrm{CH}=\mathrm{N}), 8.06(\mathrm{~s}, 1 \mathrm{H}$, Ph-H), 7.75 (d, $J=23.7 \mathrm{~Hz}, 2 \mathrm{H}, \mathrm{Ph}-\mathrm{H}), 3.33$ (s, 3H, NCH 3 ), 3.19 (s, 3H, $\left.\mathrm{CH}_{3}\right), 2.93$ (s, $\left.3 \mathrm{H}, \mathrm{CH}_{3}\right)$.

2-Chloro-5-(methylamino)benzenesulfonamide 7. ${ }^{1} \mathrm{H}$ NMR (400 MHz, DMSO) $\delta 7.40$ (s, 2H, NH $\mathrm{NH}_{2}, 7.21(\mathrm{~d}, J=44.3 \mathrm{~Hz}, 2 \mathrm{H}, \mathrm{NH}, \mathrm{Ph}-\mathrm{H}), 6.67$ (s, 1H, Ph-H), 6.29 (s, 1H, $\mathrm{Ph}-\mathrm{H}), 2.68$ (s, 3H, $\mathrm{CH}_{3}$ ). 
Ethyl (4-chloro-3-(((ethoxycarbonyl)amino)sulfinyl)phenyl)(methyl)carbamate 8. ${ }^{1} \mathrm{H}$ NMR (400 MHz, DMSO) $\delta 12.52$ (s, 1H, SO $\left.{ }_{2} \mathrm{NHCO}\right), 8.04$ (s, 1H, Ph-H), 7.67 (s, 2H, Ph-H), $4.12\left(\mathrm{q}, J=7.1 \mathrm{~Hz}, 2 \mathrm{H}, \mathrm{OCH}_{2} \mathrm{CH}_{3}\right), 4.02\left(\mathrm{q}, J=7.1 \mathrm{~Hz}, 2 \mathrm{H}, \mathrm{OCH}_{2} \mathrm{CH}_{3}\right), 3.27$ $\left(\mathrm{s}, 3 \mathrm{H}, \mathrm{NCH}_{3}\right), 1.19\left(\mathrm{t}, J=9.3 \mathrm{~Hz}, 3 \mathrm{H}, \mathrm{OCH}_{2} \mathrm{CH}_{3}\right), 1.07(\mathrm{t}, J=7.1 \mathrm{~Hz}, 3 \mathrm{H}$, $\left.\mathrm{OCH}_{2} \mathrm{CH}_{3}\right)$.

Tert-butyl (4-chloro-3-sulfamoylphenyl)carbamate 9. ${ }^{1} \mathrm{H}$ NMR (400 MHz, DMSO) $\delta$ 11.98 (s, 1H, HN-BOC), 9.87 (d, $J=12.5 \mathrm{~Hz}, 1 \mathrm{H}, \mathrm{Ph}-\mathrm{H}), 8.41$ (d, $J=11.5 \mathrm{~Hz}, 1 \mathrm{H}$, Ph-H), $7.76-7.41$ (m, 3H, Ph-H, $\left.\mathrm{SO}_{2} \mathrm{NH}_{2}\right), 1.48\left(\mathrm{~s}, 9 \mathrm{H}, \mathrm{C}\left(\mathrm{CH}_{3}\right)_{3}\right)$.

Tert-butyl

(E)-(4-chloro-3-(N-((dimethylamino)methylene)sulfamoyl)phenyl)carbamate $\mathbf{1 0} .{ }^{1} \mathrm{H}$ NMR (400 MHz, DMSO) $\delta 9.79(\mathrm{~s}, 1 \mathrm{H}, \mathrm{NH}), 8.29(\mathrm{~d}, J=2.0 \mathrm{~Hz}, 1 \mathrm{H}, \mathrm{CH}=\mathrm{N}), 8.24(\mathrm{~s}$, 1H, Ph-H), 7.56 (dd, $J=8.6,2.2 \mathrm{~Hz}, 1 \mathrm{H}, \mathrm{Ph}-\mathrm{H}), 7.44$ (d, $J=8.7 \mathrm{~Hz}, 1 \mathrm{H}, \mathrm{Ph}-\mathrm{H}), 3.18$ (s, 3H, $\left.\mathrm{CH}_{3}\right), 2.93\left(\mathrm{~s}, 3 \mathrm{H}, \mathrm{CH}_{3}\right), 1.48\left(\mathrm{~s}, 9 \mathrm{H}, \mathrm{C}\left(\mathrm{CH}_{3}\right)_{3}\right)$.

Tert-butyl (4-chloro-3-sulfamoylphenyl)(methyl)carbamate 11. ${ }^{1} \mathrm{H}$ NMR (400 MHz, DMSO) $\delta 9.78(\mathrm{~s}, 1 \mathrm{H}, \mathrm{Ph}-\mathrm{H}), 8.28(\mathrm{~s}, 2 \mathrm{H}, \mathrm{Ph}-\mathrm{H}), 7.52\left(\mathrm{~d}, J=46.8 \mathrm{~Hz}, 2 \mathrm{H}, \mathrm{SO}_{2} \mathrm{NH}_{2}\right)$, $3.20\left(\mathrm{~s}, 3 \mathrm{H}, \mathrm{CH}_{3} \mathrm{~N}-\mathrm{BOC}\right), 1.48\left(\mathrm{~s}, 9 \mathrm{H}, \mathrm{C}\left(\mathrm{CH}_{3}\right)_{3}\right)$.

Tert-butyl (4-chloro-3-(N-(ethoxycarbonyl)sulfamoyl)phenyl)(methyl)carbamate 12. 
${ }^{1} \mathrm{H}$ NMR (400 MHz, DMSO) $\delta 12.53(\mathrm{~s}, 1 \mathrm{H}, \mathrm{NH}), 8.01$ (d, $\left.J=1.7 \mathrm{~Hz}, 1 \mathrm{H}, \mathrm{Ph}-\mathrm{H}\right)$, $7.63(\mathrm{~s}, 2 \mathrm{H}, \mathrm{Ph}-\mathrm{H}), 4.01\left(\mathrm{q}, J=7.1 \mathrm{~Hz}, 2 \mathrm{H}, \mathrm{OCH}_{2} \mathrm{CH}_{3}\right), 3.22\left(\mathrm{~s}, 3 \mathrm{H}, \mathrm{NCH}_{3}\right), 1.42(\mathrm{~s}$, 9H, $\left.\mathrm{C}\left(\mathrm{CH}_{3}\right)_{3}\right), 1.08\left(\mathrm{t}, J=7.1 \mathrm{~Hz}, 3 \mathrm{H}, \mathrm{OCH}_{2} \mathrm{CH}_{3}\right)$.

$\mathrm{N}$-(4-Chloro-3-sulfamoylphenyl)-2,2,2-trifluoro-N-methylacetamide $13 .{ }^{1} \mathrm{H} \quad \mathrm{NMR}$ (400 MHz, DMSO) $\delta 8.05$ (s, 1H, Ph-H), 7.92 (d, J = 2.4 Hz, 2H, Ph-H), 7.67 (s, 2H, $\left.\mathrm{NH}_{2}\right), 3.25\left(\mathrm{~s}, 3 \mathrm{H}, \mathrm{CH}_{3}\right)$.

Ethyl ((2-chloro-5-(2,2,2-trifluoro- $N$-methylacetamido)phenyl)sulfonyl)carbamate 14. ${ }^{1} \mathrm{H}$ NMR (400 MHz, DMSO) $\delta 12.52$ (s, 1H, NH), 8.03 (s, 1H, Ph-H), 7.67 (s, 2H, $\mathrm{Ph}-\mathrm{H}), 4.12\left(\mathrm{q}, J=7.0 \mathrm{~Hz}, 2 \mathrm{H}, \mathrm{OCH}_{2} \mathrm{CH}_{3}\right), 3.27\left(\mathrm{~s}, 3 \mathrm{H}, \mathrm{NCH}_{3}\right), 1.22(\mathrm{t}, 3 \mathrm{H}$, $\left.\mathrm{OCH}_{2} \mathrm{CH}_{3}\right)$.

Ethyl ((2-chloro-5-(methylamino)phenyl)sulfonyl)carbamate 15. ${ }^{1} \mathrm{H}$ NMR (400 MHz, DMSO) $\delta 12.22\left(\mathrm{~s}, 1 \mathrm{H}, \mathrm{SO}_{2} \mathrm{NHCO}\right), 7.85\left(\mathrm{~s}, 1 \mathrm{H}, \mathrm{HN}-\mathrm{CH}_{3}\right), 7.31(\mathrm{~d}, J=8.7 \mathrm{~Hz}, 1 \mathrm{H}$, Ph-H), 7.24 (d, $J=2.8 \mathrm{~Hz}, 1 \mathrm{H}, \mathrm{Ph}-\mathrm{H}), 6.75$ (dd, $J=8.7,2.8 \mathrm{~Hz}, 1 \mathrm{H}, \mathrm{Ph}-\mathrm{H}), 4.04$ (q, $J$ $\left.=7.0 \mathrm{~Hz}, 2 \mathrm{H}, \mathrm{OCH}_{2} \mathrm{CH}_{3}\right), 2.69\left(\mathrm{~s}, 3 \mathrm{H}, \mathrm{HN}-\mathrm{CH}_{3}\right), 1.17\left(\mathrm{t}, J=7.1 \mathrm{~Hz}, 3 \mathrm{H}, \mathrm{OCH}_{2} \mathrm{CH}_{3}\right)$.

Ethyl ((2-chloro-5-(2,2,2-trifluoroacetamido)phenyl)sulfonyl)carbamate 16. ${ }^{1} \mathrm{H}$ NMR (400 MHz, DMSO) $\delta 12.57$ (s, 1H, HN-TFA), 11.72 (s, 1H, $\left.\mathrm{SO}_{2} \mathrm{NHCO}\right), 8.49$ (d, $J=$ $2.2 \mathrm{~Hz}, 1 \mathrm{H}, \mathrm{Ph}-\mathrm{H}), 8.04(\mathrm{dd}, J=8.8,2.3 \mathrm{~Hz}, 1 \mathrm{H}, \mathrm{Ph}-\mathrm{H}), 7.74(\mathrm{~d}, J=8.7 \mathrm{~Hz}, 1 \mathrm{H}$, 
Ph-H), $4.02\left(\mathrm{q}, J=7.0 \mathrm{~Hz}, 2 \mathrm{H}, \mathrm{OCH}_{2} \mathrm{CH}_{3}\right), 1.08\left(\mathrm{t}, J=7.1 \mathrm{~Hz}, 3 \mathrm{H}, \mathrm{OCH}_{2} \mathrm{CH}_{3}\right)$.

2-Chloro-5-(1H-pyrrole)benzenesulfonamide 18. ${ }^{1} \mathrm{H}$ NMR (400 MHz, DMSO) $\delta(\mathrm{ppm})$ $8.04(\mathrm{~d}, J=2.0 \mathrm{~Hz}, 1 \mathrm{H}, \mathrm{Ph}-\mathrm{H}), 7.83(\mathrm{dd}, J=8.6,2.1 \mathrm{~Hz}, 1 \mathrm{H}, \mathrm{Ph}-\mathrm{H}), 7.76(\mathrm{~s}, 2 \mathrm{H}$, $\left.\mathrm{SO}_{2} \mathrm{NH}_{2}\right), 7.72(\mathrm{~d}, J=8.6 \mathrm{~Hz}, 1 \mathrm{H}, \mathrm{Ph}-\mathrm{H}), 7.41(\mathrm{~s}, 2 \mathrm{H}, \mathrm{CH}), 6.34(\mathrm{~s}, 2 \mathrm{H}, \mathrm{CH})$.

Ethyl ((2-chloro-5-(1H-pyrrole)phenyl)sulfonyl)carbamate19. ${ }^{1} \mathrm{H}$ NMR (400 MHz, DMSO) $\delta(\mathrm{ppm}) 12.67\left(\mathrm{~s}, 1 \mathrm{H}, \mathrm{SO}_{2} \mathrm{NHCO}\right), 8.10$ (d, $\left.J=2.8 \mathrm{~Hz}, 1 \mathrm{H}, \mathrm{Ph}-\mathrm{H}\right), 7.96$ (dd, $J$ $=8.7,2.8 \mathrm{~Hz}, 1 \mathrm{H}, \mathrm{Ph}-\mathrm{H}), 7.79(\mathrm{~d}, J=8.7 \mathrm{~Hz}, 1 \mathrm{H}, \mathrm{Ph}-\mathrm{H}), 7.49-7.43(\mathrm{~m}, 2 \mathrm{H}, \mathrm{CH})$, $6.39-6.32(\mathrm{~m}, 2 \mathrm{H}, \mathrm{CH}), 4.02\left(\mathrm{q}, J=7.1 \mathrm{~Hz}, 2 \mathrm{H}, \mathrm{OCH}_{2} \mathrm{CH}_{3}\right), 1.07(\mathrm{t}, J=7.1 \mathrm{~Hz}, 3 \mathrm{H}$, $\left.\mathrm{OCH}_{2} \mathrm{CH}_{3}\right)$.

2-Chloro-5-(2,5-dimethyl-1H-pyrrole)benzenesulfonamide 21. ${ }^{1} \mathrm{H}$ NMR (400 MHz, DMSO) $\delta(\mathrm{ppm}) 7.82\left(\mathrm{~s}, 2 \mathrm{H}, \mathrm{SO}_{2} \mathrm{NH}_{2}\right), 7.78(\mathrm{~d}, J=8.4 \mathrm{~Hz}, 1 \mathrm{H}, \mathrm{Ph}-\mathrm{H}), 7.72(\mathrm{~d}, J=2.4$ Hz, 1H, Ph-H), 7.57 (dd, J = 8.4, 2.4 Hz, 1H, Ph-H), 5.85 (s, 2H, CH), 1.99 (s, 6H, $\left.\mathrm{CH}_{3}\right)$.

Ethyl ((2-chloro-5-(2,5-dimethyl-1H-pyrrole)phenyl)sulfonyl)carbamate 22. ${ }^{1} \mathrm{H}$ NMR (400 MHz, DMSO) $\delta(\mathrm{ppm}) 12.69$ (s, 1H, SO $\mathrm{SOHCO}_{2}, 7.85$ (d, J=8.4 Hz, 1H, Ph-H), $7.79(\mathrm{~d}, J=2.4 \mathrm{~Hz}, 1 \mathrm{H}, \mathrm{Ph}-\mathrm{H}), 7.70(\mathrm{dd}, J=8.4,2.4 \mathrm{~Hz}, 1 \mathrm{H}, \mathrm{Ph}-\mathrm{H}), 5.86(\mathrm{~s}, 2 \mathrm{H}, \mathrm{CH})$, $4.03\left(\mathrm{q}, J=7.1 \mathrm{~Hz}, 2 \mathrm{H}, \mathrm{OCH}_{2} \mathrm{CH}_{3}\right), 1.99\left(\mathrm{~s}, 6 \mathrm{H}, \mathrm{CH}_{3}\right), 1.09(\mathrm{t}, J=7.1 \mathrm{~Hz}, 3 \mathrm{H}$, $\left.\mathrm{OCH}_{2} \mathrm{CH}_{3}\right)$. 